\title{
Activated nano-carbon gel-electrolyte supercapacitor
}

\author{
Asheesh Kumar ${ }^{1,3}$, Kamal K. Kar ${ }^{1,2 *}$ and Malay K. Das ${ }^{3}$ \\ ${ }^{1}$ Advanced Nanoengineering Materials laboratory, Materials Science Programme, \\ ${ }^{2}$ Advanced Nanoengineering Materials laboratory, Department of Mechanical Engineering, \\ ${ }^{3}$ Energy Conversion and Storage Laboratory, Department of Mechanical Engineering, \\ Indian Institute of Technology Kanpur, Kanpur-208016, India \\ *Email: kamalkk@iitk.ac.in
}

Flexibility is the dire need of present electronics to attain the compactness at a substantial level. For that a zero dimensional material is the best material suited, and nano-powders are the closest neighbors to zero dimensional materials. Capacitor to supercapacitor transition involves specific capacity increment from microfarad to $100 \mathrm{~s}$ of farad with increasing compactness, in addition to higher energy density at the cost of considerably lower power density. In the present study activated leather grade nano-carbon $(\mathrm{N}-330)$ has been used after chemical activation at $800{ }^{\circ} \mathrm{C}$ under nitrogen atmosphere, possessing large specific surface area, suitable for fabrication of electrodes of electric double layer capacitors (EDLCs) as active material without any binder or additional conducting agent [1]. Further gel electrolyte has been used in the EDLC that adds more freedom for flexibility and compactness by avoiding leakage problem of liquid electrolytes making supercapacitor more safe and reliable. For evaluating electrochemical performance, EDLCs are tested in two electrode set-up consisting of stainless current collectors coated with activated nano-carbon and whatman ${ }^{\circledR}$ filter paper wet in $\mathrm{LiCl} / \mathrm{PVA}$ as electrolyte [2].

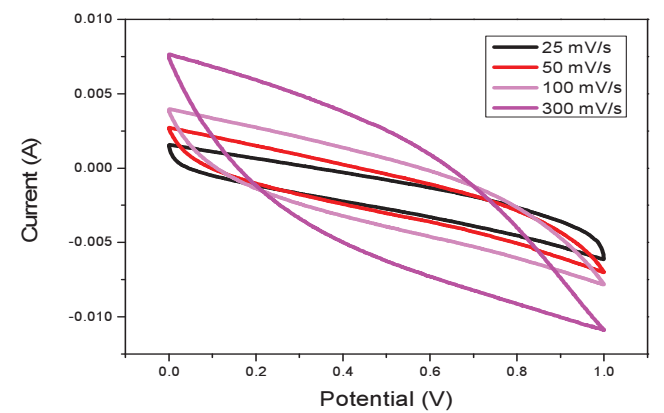

Figure 1: Cyclic voltammetry curves at different scan rates.

Figure 1 shows the cyclic voltammetry curves at different scan rates and scanned between 0 -
$1 \mathrm{~V}$ showing EDLC behavior. The galvanostatic charge/discharge cycling of nano-carbon gel electrolyte EDLC shows stable cyclic performance upto 30000 cycles and possibly beyond. Nyquist and bode plot shows the impedance and supercapacitor phase behavior after first and 30000 cycles (figure 2). The results show higher cycling stability and capacity of supercapacitor.

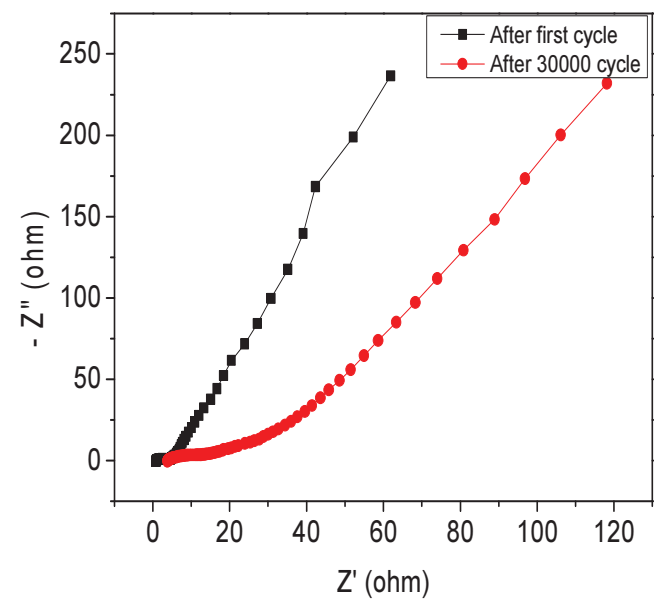

Figure 2: Nyquist and bode plots after first and 30000 cycles

\section{References}

1. W. Xu, B. Mu, A. Wang, RSC Adv., 6(9) (2016) $6967-6977$.

2. G. Wang, X. Lu, Y. Ling, T. Zhai, H. Wang, Y. Tong, and Y. Li, ACS Nano, 6(11) (2012) 10296 10302. 